\title{
Dynamics of the Leonid Meteoroid Stream: a Numerical Approach
}

\author{
P. Brown and J. Jones \\ Department of Physics, University of Western Ontario, London, \\ Ontario N6A $3 K^{7}$ Canada
}

\begin{abstract}
We have simulated the evolution of the Leonid stream via numerical integration of 3 million test particles ejected from $55 \mathrm{P} / \mathrm{Tempel}-$ Tuttle during five perihelion passages of that comet. Using the Whipple ejection velocity formula and a random ejection spread in true anomaly about the parent comet orbit inside 2.3 AU, we have followed the subsequent evolution of Leonid meteoroids differing by over 5 orders of magnitude in mass under the influence of radiation pressure and planetary perturbations. By comparing the model predictions of Leonid activity on a year by year basis with the available observations we have attempted to determine roughly the time of ejection associated with each Leonid storm occurrence and model the observed mass distribution. On the basis of the demonstrated accuracy of the model we make predictions regarding tirnes of peak activity and relative strengths for the Leonid returns for each year during the latter part of the 1990s.
\end{abstract}

\section{Introduction}

Among the meteor showers active throughout the last few centuries, none have provided displays as spectacular as those associated with the Leonid stream. The meteor storm associated with the 1833 return of the shower provided much of the stimulus for the modern study of meteors. The aura of Leonid storms is further enhanced by the streams unpredictability. While displays in 1833 and 1866-1867 were strong, the much anticipated 1899 return of the stream lead to bold predicitions all of which fell short (Olivier, 1925). Modern attempts to predict activity have relied primarily on empirical or analytic techniques to probe the stream. Yeomans' (1981) empirical study of past Leonid storms showed that most meteoroids evolved to positions spatially outside and temporally behind 55P/Tempel-Tuttle. Wu and Williams (1992) confirmed this result by simulating the stream's evolution with a small number of test particles.

Using observed orbital parameters alone to distinguish particle populations of different ages within the stream and hence infer the time between particle release and storm occurrence is not possible due to the high velocity of the Leonids and the associated large errors in the orbital parameters, even from photographic observations. Data near the time of major Leonid storms over the last century are almost exclusively in the form of visual rates. Consequently we have chosen to use the observed level of Leonid activity as the observational input constrain- 
ing our numerical model for the ejection of meteoroids from 55P/Tempel-Tuttle. This is similar to the technique used by Williams and $\mathrm{Wu}(1993,1995)$ for both the Perseids and the Giacobinids.

\section{The Model}

The observed I seonid storms are simulated by material ejected during several perihelion passages of $55 \mathrm{P} / \mathrm{Tempel}-\mathrm{Tuttle}$. As a result, we choose to sum the particle population consisting of material ejected at the five previous passages of the comet for each epoch of interest. For each passage we eject 10000 particles in each mass categrory between $1 \mathrm{~g}$ and $10^{-5} \mathrm{~g}$ in intervals of $0.1 \mathrm{in} \log _{10} \mathrm{~m}$. Thus for each epoch, the five previous passages of the comet yield $50 \times 10000 \times 5=2.5 \times 10^{6}$ ejected particles in total.

For each particle, the heliocentric distance of ejection is selected over the full cometary arc extending from $2.3>r>q$, randomly distributed in true anomaly. The radius for 55P/Tempel-Tuttle is taken to be $2 \mathrm{~km}$ after Sekanina (1972), though this estimate is highly uncertain. The cone ejection angle is taken to be $6)^{\circ}$ Here we use $\rho=800 \mathrm{kgm}^{-3}$ for the meteoroid density and take the ejection velocity for a sphere as given by Whipple (1951). This velocity coupled with the initial ejection location then forms the initial orbit for a test particle which is integrated under the influence of all planets from Venus-Neptune (excluding Mars), radiation pressure and Poynting-Robertson drag.

Each particle is followed to its descending node. It is taken to be a valid Leonid meteoroid capable of intersecting the Earth if the node occurs within $2 \times 10^{-3} \mathrm{AU}$ of the Earth's orbit at the nodal longitude of the comet. The time of nodal passage and nodal distance are recorded for the subset of particles which fulfill this condition.

\section{Results}

In the model we assume that the activity of 55P/Tempel-Tuttle has been constant during the last 500 years and ejects equal numbers of meteoroids at all mass categories. In this way the final distributions represent a normalized probability that any one particle of a given mass will intersect the Earth. To derive a final value for absolute shower activity, we must convolve this transfer-probability distribution with the absolute dust production at the comet. This involves a time-varying component (the cometary activity) and knowledge of the massdistribution throughout the coma of the comet. Unfortunately, both of these values are poorly known so for the present work we confine our analysis to the transfer efficiencies and compare the distributions to those actually observed.

The general trend of storm returns during 1833 (in particular), 1866, 1867, and 1966 is reproduced. The years where no storms occurred such as 1899 and 1933 are also represented as such. These results are shown in Figure 1. This gives us confidence that the model incorporates the essential physical processes involved in the evolution of the Leonids. In particular, we are able to reproduce the peak activity of the stream in 1966 to within 45 minutes of the observed peak and over the range of observed magnitudes. 


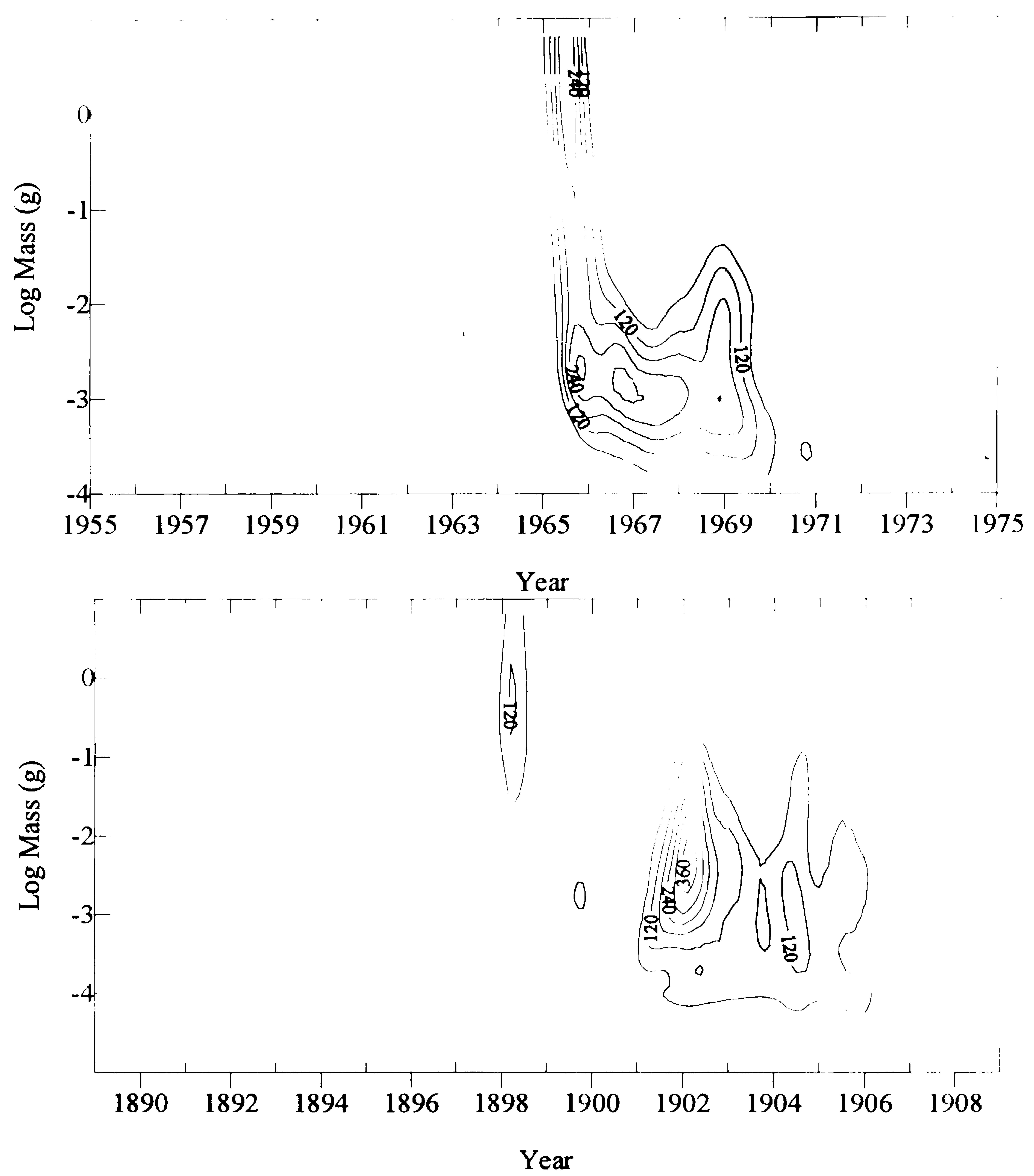

Figure 1. Number of Leonid meteoroids intersecting the Earth in bins of 0.1 in $\log _{10}$ mass and 0.1 years in time. The top plot shows the number of test meteoroids which intersected the Earth during the 1965 Leonid epoch including ejecta from perihelion passages of $55 \mathrm{P} / \mathrm{Tempel}-$ Tuttle from 1799-1932 (the five previous perihelion passages of the comet). The lower plot shows the same for the 1898 Leonid epoch. In these simulations the radius of $55 \mathrm{P} /$ Tempel-Tuttle is taken to be $2 \mathrm{~km}$, a meteoroid density of $0.8 \mathrm{~g} \mathrm{~cm}^{-3}$ is assumed and the ejection cone angle is taken to be $60^{\circ}$. 
One curious result is that most of the material responsible for Leonid storms is one or two revolutions old at most. Activity prior to five revolutions back has almost no effect on the strongest Leonid activity observed during any cycle.

The most striking result from the model is the general tendency for large numbers of smaller-sized particles to intersect the Earth for 4-5 years after $55 \mathrm{P} /$ Tempel-Tuttle's perihelion passage. This is in contrast to the observational record which suggests that strong activity in one or two years can be followed by years of dormancy. This may be due to varying activity of the parent comet or observational biases. Based on an analysis of recorded past returns, it seems most probable that the observational record, even during the 1960's, is quite incomplete. In many cases visual observations of the shower are made during less than $30 \%$ (weather conditions permitting) of any one day, and radar observations were only available from the last passage of the comet.

For the upcoming Leonid returns, the present model suggests that strong activity from the shower will begin in 1997 and continue for at least five to six years with the returns of 1998-2000 being the strongest. The material causing these storms was ejected during the 1932 and 1898 passage of the comet and is quite densely concentrated in all years about nodal longitude $235^{\circ} .16 \pm .04^{\circ}(2000.0)$. These are when we expect the maximum Leonid activity to occur, if the current version of the model truly represents the stream.

\section{Conclusions}

Our model has shown reasonable agreement with the observational record, though the latter is felt to be very incomplete. For this reason it is useful to make detailed observations of the shower during the coming return and compare the results to these model predictions. A more detailed account of this work is to be presented elsewhere (Brown and Jones, 1996).

\section{References}

Brown, P and Jones., J. 1996 Icarus, submitted.

Olivier, C.P i 925 Meteors, Williams and Wilkins, Baltimore, 1925

Sekanina, Z. 1972, in Asteroids, Comets, Meteoric Matter, C. Cristescu, Klepczynski, W.J., \& Milet, B. (eds), IAU Colloquium 22, Nice, 239.

Whipple, F。 L. 1951 AJ, 113, 464

Williams, I. \& Wu, Z. 1993, MNRAS, 264, 980

Williams, I. \& Wu, Z. 1995, Planet. Sp. Sci., 43, 723

Wu. Z. \& Williams, I. 1992, in Asteroids, Comets, Meteors 1991, A.W. Harris \& E. Bowell, LPI: Houston, 1992, 661

Yeomans, D. K. 1981, Icarus, 47, 492 